\title{
Analysis of MPV dashboard using solidworks and ABAQUS.
}

\begin{abstract}
The study is intended to measure the reliability of MPV dashboard to endure interior impact by using finite element analysis (FEA) method. A model which represents MPV dashboard was made using Solidworks and the impact simulation was done using ABAQUS. Current material being used for dashboard is ABS-GF40 and this material was proven to be reliable to withstand interior impact through the simulation that has been done in this study. Besides, this study also serve the purpose to reduce manufacturing cost using alternative materials with no less reliability compared to ABS-GF40. Similar simulation routines were applied for ABS copolymer, PP copolymer, and PVC copolymer. The simulation results showed that ABS-CF40 performance is similar to ABS-GF40, whereas the PP-CF10, PP-GF10, and PVCGF30 exhibit almost identical behavior under impact loading. Cost assessment was carried out for all materials to assist in selecting alternative material for replacement of ABS-GF40. Hence, it was found that PP-GF10 can be used as an alternative material with cost reduction up to RM33.17 on each dashboard.
\end{abstract}

Keyword: Impact analysis; Simulation; ABAQUS. 